\title{
THERMAL ANALYSIS OF UNUSUAL LOCAL-SCALE FEATURES ON THE SURFACE OF VESTA
}

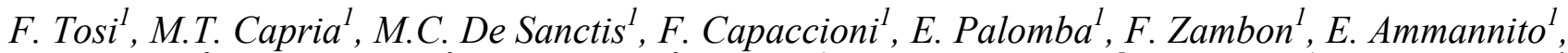 \\ D.T. Blewett ${ }^{2}$, J.-Ph. Combe ${ }^{3}$, B.W. Denevi ${ }^{2}, J .-Y$. Li $^{4}$, D.W. Mittlefehldt ${ }^{5}$, E. Palmer ${ }^{4}$, J.M. Sunshine ${ }^{6}$, \\ T.N. Titus ${ }^{7}$, C.A. Raymond ${ }^{8}$, C.T. Russell ${ }^{9}$
}

${ }^{1}$ INAF-IAPS, Via del Fosso del Cavaliere 100, I-00133 Rome, Italy, federico.tosi@iaps.inaf.it. ${ }^{2}$ The Johns Hopkins University Applied Physics Laboratory, Laurel, MD, USA. ${ }^{3}$ Bear Fight Institute, Winthrop, WA, USA. ${ }^{4}$ Planetary Science Institute, Tucson, AZ, USA. ${ }^{5}$ NASA Johnson Space Center, Houston, TX, USA. ${ }^{6}$ University of Maryland at College Park, MD, USA. ${ }^{7}$ USGS Astrogeology Science Center, Flagstaff, AZ, USA. ${ }^{8}$ NASA/Jet Propulsion Laboratory and California Institute of Technology, Pasadena, CA, USA. ${ }^{9}$ University of California at Los Angeles, Los Angeles, CA, USA.

\section{ABSTRACT}

At $525 \mathrm{~km}$ in mean diameter, Vesta is the second-most massive object in the main asteroid belt of our Solar System. At all scales, pyroxene absorptions are the most prominent spectral features on Vesta and overall, Vesta mineralogy indicates a complex magmatic evolution that led to a differentiated crust and mantle [1].

The thermal behavior of areas of unusual albedo seen on the surface at the local scale can be related to physical properties that can provide information about the origin of those materials. Dawn's Visible and Infrared Mapping Spectrometer (VIR) [2] hyperspectral images are routinely used, by means of temperature-retrieval algorithms, to compute surface temperatures along with spectral emissivities.

Here we present temperature maps of several local-scale features of Vesta that were observed by Dawn under different illumination conditions and different local solar times.

Index Terms- Asteroids, Remote sensing, Infrared spectroscopy, Surface temperature, Thermal properties.

\section{INTRODUCTION}

Localized dark and bright materials, often with extremely different albedos, were found on Vesta's surface. The range of albedos is among the largest observed on Solar System rocky bodies [3]. Evidence for mineralogical stratigraphic layering was observed on crater walls and in ejecta [1]. Dark materials, often associated with craters, appear in ejecta and crater walls, and their pyroxene absorption strengths are correlated with material brightness. It is plausible that enough dark material to match what we observe could be delivered on Vesta by low albedo (probably carbonaceous) asteroids over time [3]. The identity of the bright endmember is probably the intrinsic Vesta basaltic soil, rich in unaltered, crystalline pyroxenes [3]. It has been argued that, for the most part, Vesta's surface material can actually be thought of as having two spectral components - the dark and bright endmember spectra-in different proportions [3].

Pitted terrain is characterized by irregular rimless depressions found in and around some impact craters on Vesta, with a distinct morphology not observed on other airless bodies [4]. Similar terrains are associated with numerous Martian craters, where pits are thought to form through degassing of volatile-bearing material heated by the impact [4]. The thermal behavior of pitted terrains found on Vesta can be related to physical properties that may provide some information about the origin of those materials. VIR hyperspectral images can be used to retrieve surface temperatures, with high accuracy $(<1 \mathrm{~K})$ as long as temperatures are greater than $\sim 180 \mathrm{~K}$, which constrains this analysis to observations of the illuminated surface.

Bright and dark surface materials on Vesta, as well as pitted materials, are currently being investigated by the Dawn team $[3,4]$. We present temperature maps and spectral profiles of some of these features as derived from VIR data in different phases of the mission. Such information may constrain their thermophysical properties and provide insight on their composition and physical structure.

\section{DATA SET AND ANALYSIS}

After the initial Survey Orbit (11 through 31 August 2011) and High Altitude Mapping Orbit (HAMO-1, 29 September through 1 November 2011), Dawn spiraled down to its 210$\mathrm{km}$ above mean surface Low Altitude Mapping Orbit (LAMO), which was by far the longest phase of the Vesta mission (12 December 2011 through 30 April 2012). The Dawn spacecraft eventually raised its altitude back to 685 $\mathrm{km}$ above mean surface to perform a second High Altitude Mapping Orbit (HAMO-2, 15 June to 25 July 2012), prior to 
its departure from Vesta. During Dawn's one-year mission at Vesta, the heliocentric distance of the asteroid increased from 2.23 to 2.55 Astronomical Units (AU) - the latter value being close to aphelion.

Data acquired by the Framing Camera (FC) in the Survey phase first revealed the existence of several unusual surface features: 1) bright and dark material deposits, 2) spectrally distinct ejecta, 3 ) regions suggesting finer-grained materials. Terrains with a distinct pitted morphology were identified later, on the basis of FC clear-filter images obtained during LAMO [4].

Here we calculate surface temperatures of several localscale features, particularly dark and bright material deposits and pitted terrains, using VIR data acquired especially in the Survey and High Altitude mapping orbits. In the Survey phase, VIR obtained resolved hyperspectral images of more than $65 \%$ of the surface of Vesta with average spatial resolution of $\sim 0.68 \mathrm{~km}$. In the HAMO- 1 and -2 phases, VIR acquired hyperspectral images at a roughly constant pixel resolution of $0.17 \mathrm{~km}$. Even though footprints were mostly non-continuous due to the higher instantaneous speed of the ground tracks, some of the dark/bright material deposits and the four known pitted-terrain sites (Marcia, Cornelia, Licinia, Numisia) were re-observed in greater detail. VIR data acquired in LAMO have much reduced spatial coverage and larger phase angles (resulting in long shadows) with respect to Survey and HAMO data, and as such LAMO data will not be discussed here.

On the illuminated side of Vesta, the region of the infrared spectrum beyond $\sim 3.5 \mu \mathrm{m}$ is dominated by thermal emission from the asteroid's surface, which can be used to determine surface temperature by means of temperatureretrieval algorithms. To calculate surface temperatures, we applied a Bayesian approach to nonlinear inversion [5] based on the Kirchhoff law $r_{\lambda}=1-\varepsilon_{\text {r }}$ and the Planck function.

Results were compared with those provided by the application of alternative methods (e.g, [6]). In all cases, the minimum retrievable temperature $(\sim 180 \mathrm{~K})$ is set by the Noise Equivalent Spectral Radiance (NESR), i.e. the RMS noise of the in-flight measurements expressed in units of spectral radiance. On the other hand, for a given local solar time (LST), the maximum temperature depends on incidence angle and surface properties such as thermal inertia and albedo.

\section{RESULTS}

Some bright features of Vesta have an overall albedo in the visible as much as $40 \%$ brighter than surrounding areas. Data from the IR channel of VIR show that bright regions generally correspond to regions with lower thermal emission, i.e. lower temperature, while dark regions correspond to areas with higher thermal emission, i.e. higher temperature (Fig. 1). This behavior confirms that many of the dark appearances in the VIS mainly reflect albedo variations, and not, for example, shadowing.

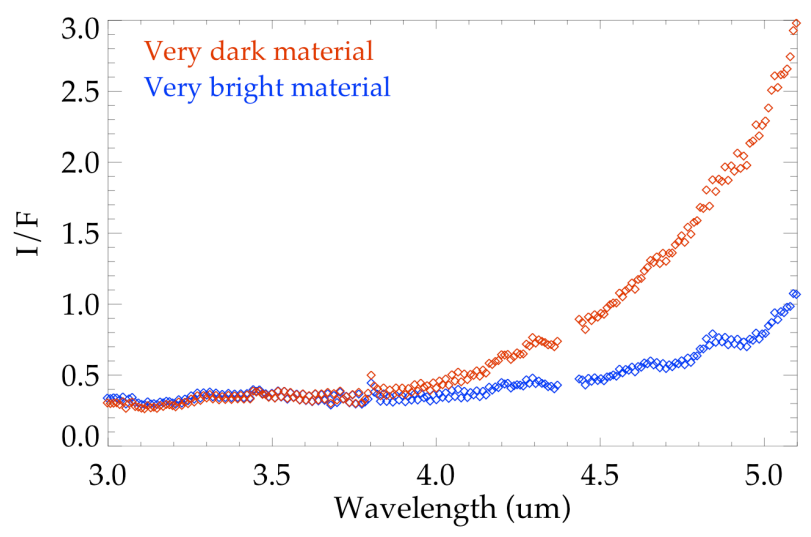

Fig. 1. Spectral comparison between very dark material (cube: 373312662 ) and very bright material (cube: 394683501) observed respectively in HAMO-1 and HAMO-2 by VIR in its infrared domain. Very dark material (red dots) shows a thermal emission significantly greater than very bright material (blue dots) observed at the same spatial scale and under similar solar illumination and local solar time.

In particular, during maximum daily insolation, dark features in the equatorial region may show temperatures greater than $270 \mathrm{~K}$ during the observed range of heliocentric distances, while bright features seen at comparable spatial resolution and local solar time are at least $10 \mathrm{~K}$ cooler. However, individual features may show different thermal behaviors due to differences in composition (revealed also by the emissivity) and/or structure (e.g, average grain size of the surface regolith, porosity, etc.).

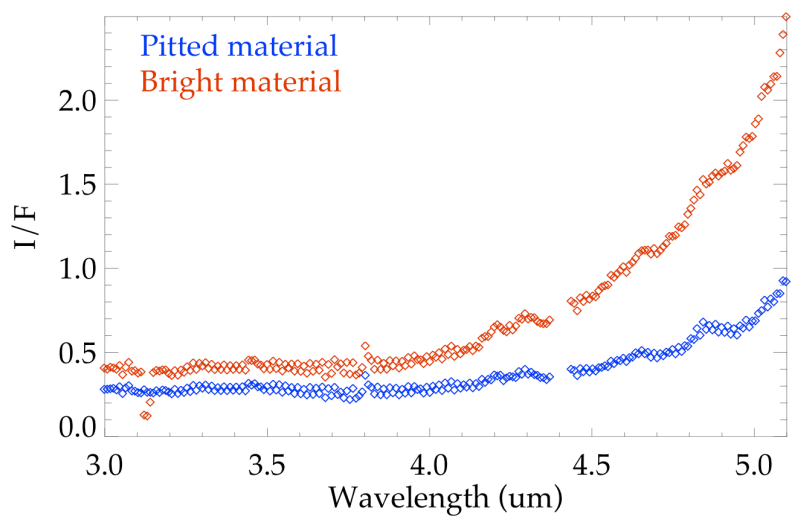

Fig. 2. Spectral comparison based on VIR infrared data between pitted terrain in crater Marcia (blue curve) and very bright material in nearby crater Calpurnia (red curve). Pitted terrain, despite its lower albedo, exhibits a significantly reduced thermal emission compared to very bright material, even under comparable solar illumination and local solar time. 
Pitted terrains display distinct thermal properties compared to other terrains on Vesta. They show a significatly reduced thermal emission at infrared wavelengths longward of $4 \mu \mathrm{m}$ (Fig. 2), have distinct margins in the temperature images, and are cooler than nearby terrains even though they show similar albedos and are observed at similar solar illumination conditions.

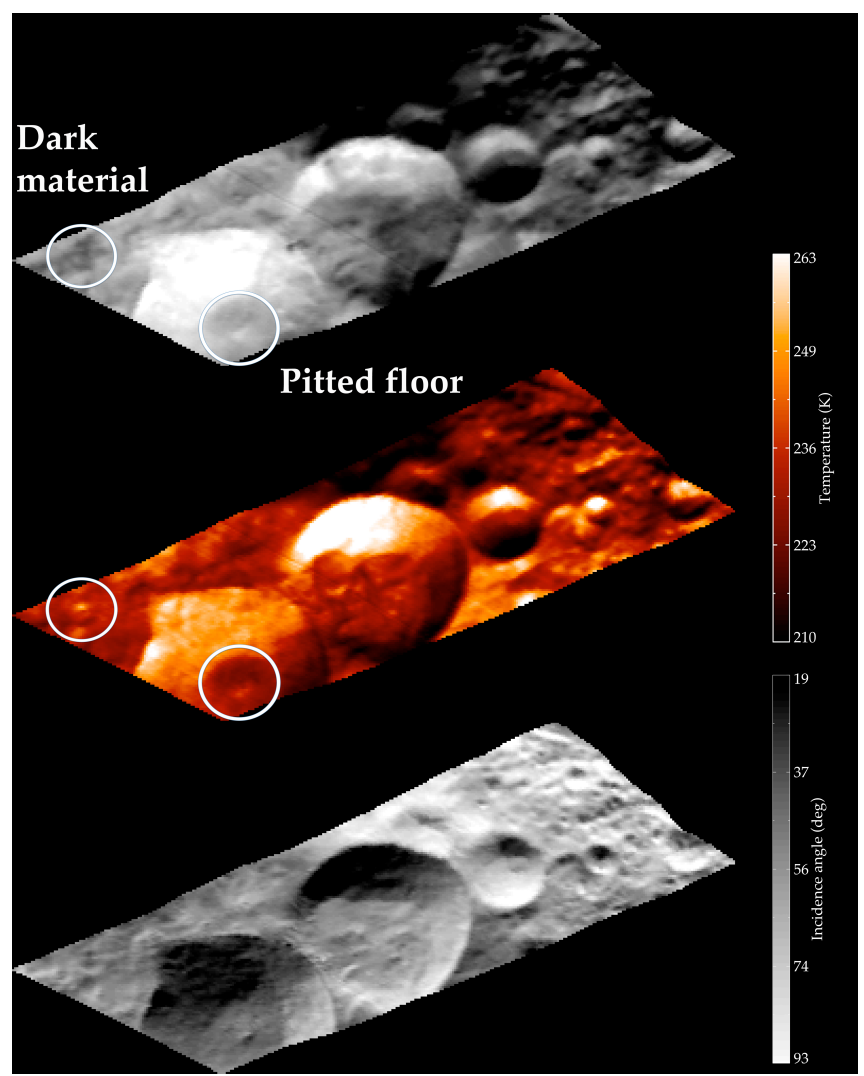

Fig. 3. Simple cylindrical projection of a portion of Marcia crater, observed by VIR (cube: 366641356$)$ during the Survey phase $(680 \mathrm{~m} / \mathrm{pixel})$. The upper panel shows the region as seen at the near-infrared wavelength of 1.4 $\mu \mathrm{m}$. The middle panel shows a temperature map of the same area, as derived from VIR infrared spectra using the method described in [5] in the range 4.5-5.1 $\mu \mathrm{m}$, where thermal emission is important. The bottom panel shows the local solar incidence angle measured from the surface normal to a detailed shape model. Materials illuminated at high incidence angles are generally cooler than materials at low incidence angles. The spectrally distinct material associated with Marcia's floor (lower left side of the projected image), observed at $10.4 \mathrm{~h} \mathrm{LST}$, is $\sim 10 \mathrm{~K}$ colder than the rest of the crater where illumination conditions are similar, and has similiar to slightly lower temperatures than Marcia's dark ejecta blanket. The average surface temperature on the crater's floor where the pitted material is located is $234 \mathrm{~K}$, lower than the average temperature of $241 \mathrm{~K}$ found on the floor of the nearby crater Calpurnia (center of the image) observed at 11.0 LST and the temperature of $\sim 260 \mathrm{~K}$ found in the northern wall of Calpurnia observed at $11.1 \mathrm{~h} \mathrm{LST}$.

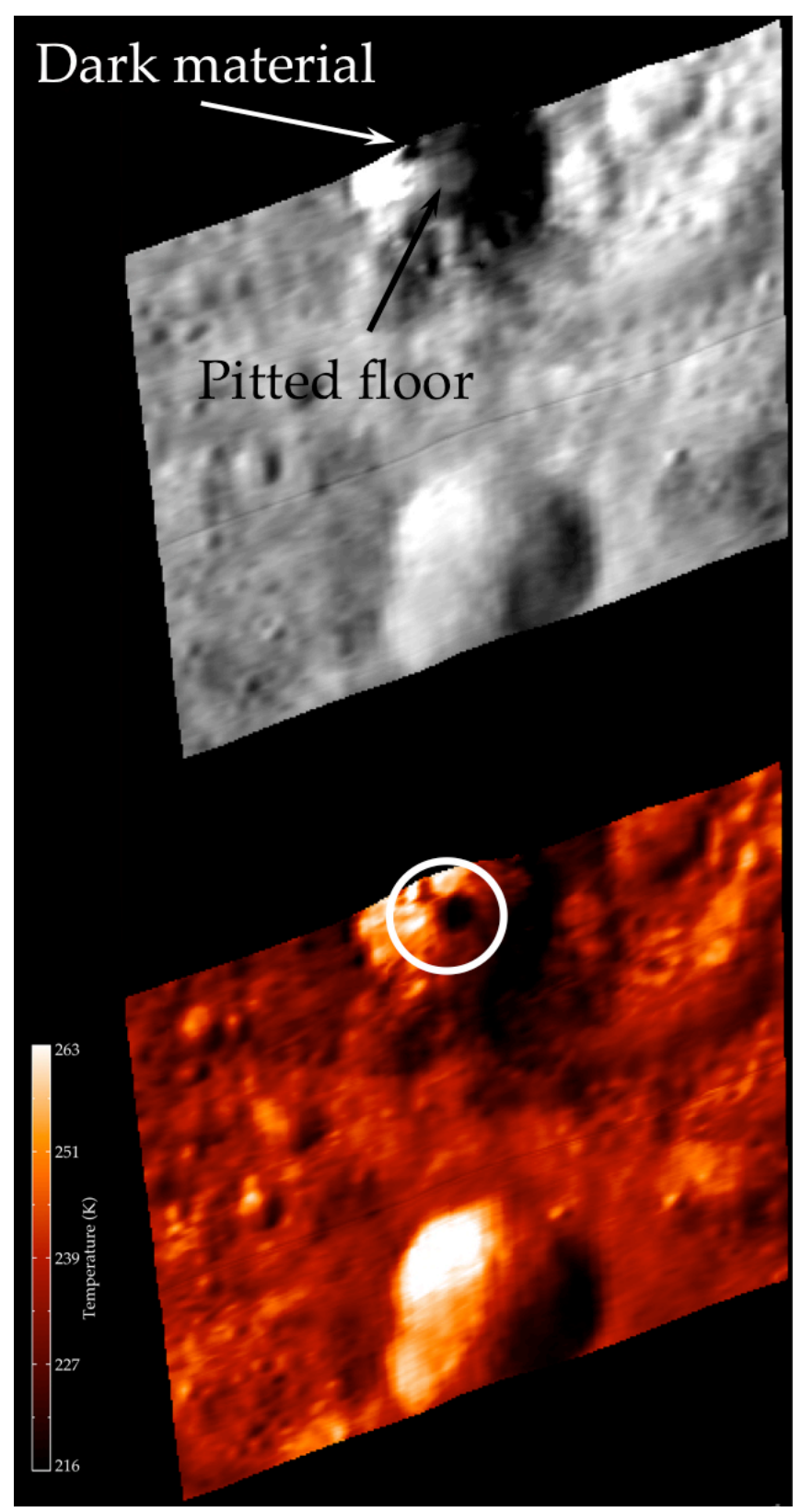

Fig. 4. Simple cylindrical projection of a portion of Cornelia crater, observed by VIR (cube: 371813008) during the HAMO-1 phase (171 $\mathrm{m} / \mathrm{pixel}$ ). The upper panel shows the region as seen at the near-infrared wavelength of $1.4 \mu \mathrm{m}$. The lower panel shows a temperature map of the same area, as derived from VIR infrared data. Pitted material associated with Cornelia's floor, observed at $9.5 \mathrm{~h} \mathrm{LST}$, has an average temperature of $229 \mathrm{~K}$ and shows distinct margins in the thermal image, clearly standing out compared to the lit portion of the crater and particularly to the dark material exposed in its northern inner wall, which has an average temperature of $256 \mathrm{~K}$. 
Pitted terrains can be $>10 \mathrm{~K}$ cooler than the surroundings, and even cooler than bright material deposits found on Vesta, despite their lower albedo (Figs. 3, 4).

Spectral emissivity profiles retrieved for pitted terrains found in the floors of Marcia and Cornelia reveal prominent differences with respect to other distinct features discovered on Vesta, most notably dark and bright material deposits (Fig. 5). These are likely related to differences in both composition and physical structure of the surface material. The lower temperatures suggest a higher thermal inertia, i.e. a slower response to insolation, which may be due to reduced local porosity (i.e., increased local density) and/or an increase in the local thermal conductivity.

These might be caused by rapid degassing of volatilebearing materials: local cavities at micrometer to centimeter scale gradually disappear, leaving a compact layer. An increase of both density and thermal conductivity may actually be responsible for the observed behavior. This is consistent with the lower albedo of compact material compared to the unconsolidated material widespread on the surface of Vesta.

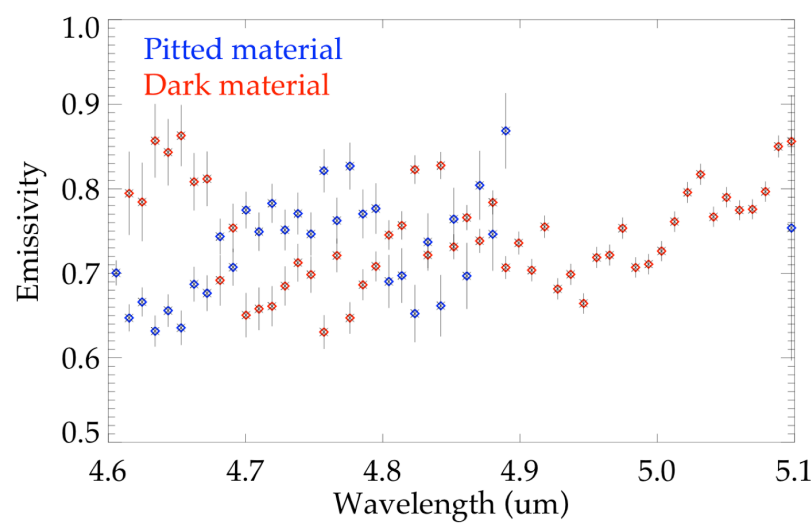

Fig. 5. Comparison of spectral emissivities, retrieved from VIR infrared data, between pitted terrain in the floor of Cornelia (blue dots) and dark material found in the wall of the same crater (red dots). The interval 4.9-5.1 $\mu \mathrm{m}$ is unsuitable for pitted material due to the superposition of solar reflectance and thermal emission, which yields no emissivity information. These two profiles reveal an anti-correlation at wavelengths shorter than $4.9 \mu \mathrm{m}$, which may be related to a difference in composition.

\section{ACKNOWLEDGMENTS}

This work was supported by the Italian Space Agency (ASI), ASI-INAF Contract I/004/12/0. Support of the Dawn Science, Instrument, Operations Teams, as well as of the Dawn at Vesta Participating Scientist program, is gratefully acknowledged. We warmly thank Robert Gaskell (PSI) for providing a detailed shape model of Vesta that was used in this work.

\section{REFERENCES}

[1] De Sanctis, M.C., et al. (2012), "Spectroscopic characterization of mineralogy and its diversity across Vesta". Science 336, pp. 697-700.

[2] De Sanctis, M.C., et al. (2011), "The VIR Spectrometer". Space Science Reviews 163 (1-4), pp. 329-369.

[3] McCord, T.B., et al. (2012), "Dark material on Vesta from the infall of carbonaceous volatile-rich material”. Nature 491 (7422), pp. 83-86.

[4] Denevi, B.W., et al. (2012), "Pitted terrain on Vesta and implications for the presence of volatiles". Science 338, pp. 246249.

[5] Keihm, S., et al. (2012), "Interpretation of combined infrared, submillimeter, and millimeter thermal flux data obtained during the Rosetta fly-by of Asteroid (21) Lutetia”. Icarus 221, pp. 395-404.

[6] Clark, R.N., et al. (2011), "Thermal removal from near-infrared imaging spectroscopy data of the Moon". Journal of Geophysical Research 116, CiteID E00G16. 